\title{
MedSim: A Novel Semantic Similarity Measure in Bio-medical Knowledge Graphs ${ }^{\star}$
}

\author{
Kai Lei ${ }^{[0000-0001-9197-895 X]}$, Kaiqi Yuan ${ }^{[0000-0003-0985-3790]}$, Qiang \\ Zhang ${ }^{[0000-0003-3922-2789]}$, and Ying Shen区[0000-0002-3220-904X] \\ School of Electronic and Computer Engineering, \\ Peking University Shenzhen Graduate School, Shenzhen, China \\ \{leik,shenying\}@pkusz.edu.cn, kqyuan@pku.edu.cn, zhangqiang@sz.pku.edu.cn
}

\begin{abstract}
We present MedSim, a novel semantic SIMilarity method based on public well-established bio-MEDical knowledge graphs (KGs) and large-scale corpus, to study the therapeutic substitution of antibiotics. Besides hierarchy and corpus of KGs, MedSim further interprets medicine characteristics by constructing multi-dimensional medicine-specific feature vectors. Dataset of 528 antibiotic pairs scored by doctors is applied for evaluation and MedSim has produced statistically significant improvement over other semantic similarity methods. Furthermore, some promising applications of MedSim in drug substitution and drug abuse prevention are presented in case study.
\end{abstract}

Keywords: Semantic similarity - Semantic networks · Bioinformatics.

\section{Introduction}

Semantic similarity metric is widely used in medical information retrieval [1] and medical knowledge reasoning. The most promising application scenario is therapeutic substitution, also known as therapeutic interchange and drug substitution. It is the practice of replacing one prescription with chemically different drugs that are expected to have the same clinical effect. Medicine semantic similarity measure plays an important role in this context by enabling a proper interpretation of drug information [2].

Unlike conventional similarity measures, semantic similarity methods based on Knowledge Graph (KG) have been proven effective in nature language processing and information retrieval $\underline{3}$. KG is a type of graph structure that records massive entities and relations, such as FreeBase [4] and DrugBank 5]. DrugBank is a well-known bioinformatics KG for its broad scope and great integrity, which classifies medicines with multi-category bio-medical knowledge bases.

The existing KG-based semantic similarity can be classified into structurebased similarity measures, and corpus-based ones. Aimed at heterogeneous networks, PathSim [6, and random walk 7] are introduced to compute semantic

\footnotetext{
* Supported by the National Natural Science Foundation of China (No.61602013) and the Shenzhen Key Fundamental Research Projects (Grant No. JCYJ20170818091546869 and JCYJ20170412151008290).
} 
similarity among the same type of objects by fully utilizing the path information. IC-based measures primarily rely on the contextual information of words, which usually measures the general semantic relevance between two words 88. Distributed representation methods 9] calculate semantic similarity by transforming concepts into dense low-dimensional vectors learned from the large scale of corpus. Hybrid measures combine structural features with corpus features to overcome data sparseness and data noise. A weighted path method (Wpath) 10] is proposed by employing both path length and information content. SimCat [1] incorporates category corpus and relationship structure information. However, due to the limited coverage of KGs, the aforementioned measures cannot be directly applied to a specific domain.

The bio-medical field has complex and diverse terminology, hierarchies and attributes to be considered. Pedersen et al. 12 presented a cluster-based approach with new features and evaluated this method for two different bioinformatic knowledge bases within the UMLS. Traverso et al. 13 proposed GADES to compare entities in bioinformatic knowledge graphs by encoding the KG in aspects, e.g., hierarchies, neighborhoods, and specificity. Even though these semantic simlarity methods consider the characteristics in the bio-medical field, they often rely on a limited number of data sources and are validated in a limited scale of dataset. Besides, they exclusively depend on the KG-mined features rather than fully utilized the textual information of KG.

To address the problem above, we propose MedSim, a novel semantic similarity method based on public well-established medicine KG and multi-category data sources, to study the therapeutic substitution of antibiotics. Antibiotics are extensively applied as antimicrobial agents in disease treatment but the abuse of antibiotics is becoming increasingly serious. We consider the semantic similarity between two antibiotics in bio-medical category using not only medicine-specific features but also the structure and corpus information of DrugBank which is freely accessible. To our knowledge, the biomedical domain never witnesses the standard human rating datasets for semantic similarity publications. A dataset labeled by doctors which is much larger than other medical similarity methods 14, 15 is applied to evaluate MedSim. To make our method more reproducible, the dataset is freely accessible in Github 11. The main contributions of this work can be summarized as followed:

- To improve the interpretability of drug property and the context-based word representation, the one-dimensional vector of medicine-specific features is transformed to multi-dimensional weighted vectors. The medicine-specific features reflect medicine characteristics and can be extended to all types of medicine.

- To reduce the noises introduced by hierarchical structure on semantic similarity metrics, we employ a KG-based hierarchy embedding feature and corpus-based semantic-level features, of which the combination can mine more information of interest and simplify the labor-intensive process compared with universal fields.

\footnotetext{
${ }^{1}$ https://github.com/YuanKQ/MedSim-antibiotics-labeled-dataset
} 
- Experiment results show that compared with the existing methods, MedSim can evaluate similarity more effectively. This reveals that on the analytics and assessments of semantic network, domain specific features, structural and textual information are important.

The rest of this paper is organized as follows: Section 2 presents the process of dataset. Section 3 proposes our semantic similarity method MedSim based on bio-medical KGs. Section 4 reports the evaluation experiments and explains the evaluation results. Conclusions and future work are outlined in Section 5 .

\section{Data Processing}

\subsection{Preparation of Data Source}

Semantic similarity measures relied on single data source provide only partial information about a subset of interest and the computed results show various degree of incompleteness. To address this problem, MedSim integrates the following well-established and widely used multi-category data sources.

DrugBank DrugBank [5] is a comprehensive bioinformatics and cheminformatics KG that combines detailed drug entities with drug information. It contains 10,513 drug entities including 1,739 approved small molecule drugs, 873 approved biotech (protein/peptide) drugs, 105 nutraceuticals and over 5,029 experimental drugs. Each drug entity contains more than 200 properties, such as chemical structure, prescription, pharmacology, pharmacoeconomics, spectra, etc.

SIDER SIDER [16] is a side effect database of information on marketed medicines and their recorded adverse drug reactions, including 1430 drugs and 5868 side effects. The relationships between antibiotics and the corresponding side effects will be extracted to calculate the side effect based similarity in MedSim.

NDF-RT National Drug File Reference Terminology (NDF-RT) [17] combines the hirerachical drug classification with multiple drug characteristics including physiologic effect, mechanism of action, pharmacokinetics, etc. We extract mechanism of the essential pharmacologic properties of medications (physiologic effect and mechanism of action) from NDF-RT.

PubMed PubMed [18] is a bio-medical search engine accessing more than 27 million citations for biomedical literature from MEDLINE, life science journals, and online books. We crawl more than 500,000 papers about medicine via the PubMed API to help establishing the semantic features of MedSim. 


\subsection{Antibiotic Pairs Labeling}

To verify the effectiveness of MedSim, we conduct experiments on 52 most commonly used antibiotics of 10 categories in hospital. With the combination of these antibiotics, 1326 pairs are generated. 528 randomly selected pairs cover nearly $40 \%$ of the total. Referring to [19,20], doctors, from the perspective of clinical application, score the similarity between two antibiotics, which ranges in $[0,1]$, according to both antibacterial spectrum and efficacies of medicine. 0 indicates that there is no similarity between two antibiotics, while 1 implies that the two antibiotics are extremely similar. The adverse reactions, side effects, patients past history and other factors are left aside in this stage. To make antibiotic pairs labeling more accurate, each pair is labeled by at least 3 doctors and the average is taken as final result. The Pearson coefficient between the scores issued by each doctor and the average score ranges from $82.7 \%$ to $86.4 \%$ while Spearman coefficient ranges from $79.2 \%$ to $88.8 \%$, both proving the reliability of doctors' assessment. Scores about the antibiotic similarity were uploaded to Github.The labeled antibiotic pairs are divided into training set and test set, which will be used in our regression prediction model in Section 4 .

\section{Methodology}

MedSim is a medicine similarity metric predicted by the random forest regression model learned from the following features: medicine-specific features (side effect, target protein, mechanism of action, physiological effect), structure feature of concept taxonomy (hierarchy embedding-based feature), and semanticlevel features (KG-based semantic textual similarity and word embedding-based feature).

\subsection{Medicine-specific Features (MF)}

Domain-specific KGs and multi-category corpus are adopted to mine medicalspecific features, so as to address the incompleteness of drug attributes from single data source. Side effect, target protein, mechanism of action, and physiological effect are utilized to explore the medicine-specific features which can simplify the semantic representation of medicines.

Instead of simply flattening all properties into one vector, the weighted property vectors of different features from multiple data sources are generated to interpret the characteristic of drugs. For a drug, its multi-dimensional weighted feature vector is erected by stacking weighted vectors of all medicine-specific features. Each row of vector represents one category of characteristics, in which the values demonstrate the weights of specific properties. Fig. 1 1 shows a snapshot of multi-dimensional weighted feature vector of an antibiotic: nitrofurantoin.

Side Effect based Similarity For a drug d, its side effects can be obtained from SIDER database. In the paper, we want to find out side effects related 


\begin{tabular}{|c|c|c|c|c|c|c|}
\hline Side Effect & 0 & 0 & 0 & $\ldots$ & 0 & 0.545 \\
\hline Target & 0 & 0 & 0 & $\ldots$ & 7.074 & 0 \\
\hline Machamism & 0 & 3.640 & 0 & ... & 3.855 & 0 \\
\hline Physiology & L7.871 & 0 & 0 & $\ldots$ & 0 & 0 \\
\hline
\end{tabular}

Fig. 1. Part of multi-dimensional weighted feature vector of nitrofurantoin.

to some drug, as well as those specific to this certain drug, hence improve the discrimination. Inverse Document Frequency (IDF) can work well in alleviating the impact of high frequency terms and pay more attention to rare ones:

$$
\operatorname{IDF}(s, \text { Drugs })=\frac{\log (\mid \text { Drugs } \mid+1)}{\operatorname{DF}(s, \text { Drugs })+1}
$$

Where Drugs is the set of all drugs, $s$ is a side effect, and DF $(s$, Drugs $)$ is the number of drugs with the side effect sider $s$. The weighted side effect vector of a drug $d$ is sider $(d)$, consisting of side effects extracted from SIDER. The value of element $s$ of sider $(d)$, denoted sider $(d)[t]$, is $\operatorname{IDF}(s, \operatorname{Drugs})$ if it is one of the side effects of drug $d$, otherwise it is 0 . The side effect-based similarity of two drugs $d_{1}, d_{2}$ is the cosine distance of the vectors $\operatorname{sider}\left(d_{1}\right)$ and $\operatorname{sider}\left(d_{2}\right)$.

Target based Similarity The information about proteins targeted by a drug $d$ is collected from DrugBank. The target-based similarity of two drugs $d_{1}, d_{2}$ is defined as the cosine similarity of IDF-weighted target protein vectors of two drugs, which are calculated like the IDF-weighted side effect vector.

Mechanism based Similarity We collect all the mechanisms of a drug from NDF-RT. The mechanism-based similarity of two drugs is calculated by the cosine distance of IDF-weighted mechanism vectors of two drugs as mentioned in the previous paragraph.

Physiological Effect based Similarity The IDF-weighted physiological effect vectors of drugs are also established from NDF-RT, and physiological effectbased similarity measure is the same as the mechanism-based similarity.

\subsection{Hierarchy Embedding-based Feature (HF)}

To fully take advantage of taxonomy hierarchy information, DeepWalk [21] is applied to learn the hierarchy embedding from the taxonomy hierarchy in DrugBank. All concepts in taxonomy hierarchy are actually arranged as a concept graph, where nodes represent concepts and edges indicate hierarchical relations.

Unlike some structure-based methods which focus on concrete properties of KG, e.g. neighbors, class hierarchies and node degrees, hierarchy embedding based feature is able to map a total knowledge graph into a low dimension 
vector space while preserving certain properties of the original graph. Due to the limited depth of taxonomy hierarchy, the training paths in DeepWalk can cover both complete leaf to root path and neighbor nodes, which can fully utilize structural information. The hierarchy embedding-based similarity is calculated by the cosine distance between two drug vectors.

\subsection{Semantic-level Features (SF)}

We adopt two types of semantic-level features: the KG-based semantic textual similarity feature is used to process the entity related textual information, while the word embedding-based similarity feature learned from the context of drugs is used to cluster similar entities in vector space.

KG-based Semantic Textual Similarity (KSTS) Traditional IC-based semantic similarity metrics require a large domain corpus and cost-intensive labor to remove redundant data. KGs have already mined topic-related knowledge from textual corpus, which has prepared a high-quality domain corpus. The entity description or other textual information about the concepts in KG usually implies the nature of the concepts. The greater is the similarity among the concepts, the greater is the similarity of the words in their entity description.

The bio-medical proper nouns in the entity description of universal KG tend to have few information, which cannot improve the performance of the semantic textual similarity measures 22. In this context, BM25 algorithm 23] is applied to compute the textual similarity based on entity description by converting the ranking score into the similarity score that ranges between 0 and 1 .

Given a description of a drug $d_{1}$ containing the keywords $q_{1}, q_{2}, \ldots, q_{n}$, the BM25 score of a description $D$ of another drug $d_{2}$, is

$$
\operatorname{score}\left(d_{1}, d_{2}\right)=\sum_{i=1}^{n} I D F\left(q_{i}\right) \cdot \frac{f\left(q_{i}, D\right) \cdot\left(k_{1}+1\right)}{f\left(q_{i}, D\right)+k_{1} \cdot\left(1-b+b \cdot \frac{|D|}{a v g d l}\right)}
$$

where $\operatorname{IDF}\left(q_{i}\right)$ is the IDF weight of the keywords $q_{i}, f\left(q_{i}, D\right)$ is the occurrence frequency in the description $D,|D|$ is the number of words in $D$, and avgdl is the average number of words of all entity descriptions drawn from DrugBank. Usually, the free parameters $k_{1} \in[1.2,2.0]$ and $b=0.75$. Here, we set $k_{1}=2$ and $b=0.75$. To normalize the BM25 ranking score, the KG-based Semantic Textual Similarity is definded below, where Drugs is the set of all drugs:

$\operatorname{KSTS}\left(d_{1}, d_{2}\right)=\frac{\operatorname{score}\left(d_{1}, d_{2}\right)-\min \{\operatorname{score}(x, y) \mid x, y \in \operatorname{Drugs}\}}{\max \{\operatorname{score}(x, y) \mid x, y \in \operatorname{Drugs}\}-\min \{\operatorname{score}(x, y) \mid x, y \in \operatorname{Drugs}\}}$

The entity description included in DrugBank is few in words but large in numbers. Thereby, BM25 can quickly measure the textual semantic similarity between a drug and the rest. 
Textual Embedding-based Similarity Word2vec is applied to train the textual embedding vector of PubMed 500,000 indexed papers and medical corpus (e.g. DrugBank and DailyMed). We use the skip-gram model rather than CBOW according to the pre-experimental results. Since word2vec can predict concept relatedness by simple algebraic operations in vector space, the word embeddingbased similarity is calculated by the cosine similarity between vectors of corresponding drugs.

\subsection{Random Forest Regression Model}

Based on the aforementioned features, random forest regression model is applied to measure the medicines semantic similarity. Random forest is an effective ensemble learning algorithm for regression task. In this paper, random forest is used to predict the similarity of an antibiotic pair (a scalar dependent variable $y$ ) learned from the selected features of samples (explanatory variables $X$ ).

Ten-fold cross validation is applied to train the model. The training and test datasets are from the labeled antibiotic pairs mentioned in Section 3.2, except some pairs whose labeled scores substantially differ. The Root Mean Square Error (RMSE), Mean Absolute Error (MAE), Pearson correlation coefficient and Spearman rank correlation coefficient are adopted to evaluate the model. To select the best regression model, we make a detailed comparison of the random forest regression model and other common methods such as linear regression, logistic regression, polynomial regression, tree regression, etc.

As shown in Table 1, the model that we employed has the lowest RMSE and MAE, which indicates that it outperforms other models in precision and stability. The Pearson and Spearman coefficient specify that the similarity measured by the random forest has a strong correlation with the results scored by doctors. Compared to other regression models, random forest is not very sensitive to missing data, which alleviates the impact from the incompleteness of drug attributes. Besides, the randomized sampling before bagging and the application of averaging can avoid overfitting and further improve the generalization ability.

Table 1. Performance comparison of regression models.

\begin{tabular}{|l|l|l|l|l|}
\hline Regression model & Person & Spearman & RMSE & MAE \\
\hline Logistic Regression & 0.273 & 0.239 & 2.391 & 1.641 \\
Linear Regression & 0.306 & 0.232 & 0.186 & 0.137 \\
Polynomial Regression & 0.431 & 0.442 & 0.178 & 0.137 \\
Support Vector Regression & 0.456 & 0.435 & 0.175 & 0.124 \\
Adaboost Regression & 0.465 & 0.435 & 0.172 & 0.129 \\
Bagging Regression & 0.520 & 0.474 & 0.164 & 0.123 \\
Tree Regression & 0.563 & 0.510 & 0.161 & 0.116 \\
Random Forest Regression & $\mathbf{0 . 5 8 4}$ & $\mathbf{0 . 5 1 8}$ & $\mathbf{0 . 1 5 6}$ & $\mathbf{0 . 1 1 6}$ \\
\hline
\end{tabular}




\section{Evaluation}

To assess the performance of our model, we conduct two types of experiments. We first compare it with the state-of-art semantic similarity methods to prove whether MedSim outperforms others. Furthermore, we measure the prediction ability of individual features and different feature combinations to exploit the effect of the model performance. Spearman and Pearson correlations coefficients are widely used to evaluate semantic similarity measures. In this section, both coefficients are adopted to evaluate the correlation between doctor assessment and experiment results, while Z-significance test between MedSim and baselines is used to evaluate whether MedSim statistically outperforms other methods using two-sided test and 0.05 statistical significance.

\subsection{Comparison with State-of-art Similarity Metrics}

We compare MedSim with four state-of-art algorithms, including GADES [13], Res [8], Wpath [10] and Hybrids [15]. The GADES is a structure-based measure, while Res is an information content based measure. Wpath considers both path and IC information. Based on Wpath, the method Hybrids takes medical properties into account to calculate the drug similarity.

As shown in Table 2, different semantic similarity method has different level of correlation between doctors judgment and MedSim outperforms the others.

Table 2. Comparison between semantic method.

\begin{tabular}{|l|l|l|l|l|}
\hline \multirow{2}{*}{ Method } & \multirow{2}{*}{ Pearson } & \multirow{2}{*}{ Spearman } & \multicolumn{2}{|c|}{ Z significance test } \\
\cline { 4 - 5 } & & & Z statistic & p-value \\
\hline GADES & 0.251 & 0.203 & 2.881 & 0.051 \\
Res & 0.211 & 0.223 & 0.273 & 0.000 \\
Wpath & 0.251 & 0.205 & 0.805 & 0.000 \\
Hybrids & 0.256 & 0.278 & 0.995 & 0.000 \\
MedSim & $\mathbf{0 . 5 8 6}$ & $\mathbf{0 . 5 2 3}$ & N $/ \mathrm{A}$ & N/A \\
\hline
\end{tabular}

GADES has the lowest Spearman correlation, probably due to the structure of bio-medical KGs. It is common sense that in KGs, the upper-level concepts in a taxonomy are supposed to be more general hence have more entities. However, it may be different in bio-medical KGs, where the entity number of lower-level concepts would be larger than that of upper-level concepts. For example, according to concept tree in the latest released version (version 5.0.6) of DrugBank, the level of Tetracyclines is upper than that of Aminobenzene sulfonamides. However, the entity number of the former one is 3246, which is far less than that of the latter one, which has 235515 entities. Thereby, structure based approach like GADES cannot work well in the bio-medical KG-based similarity measures. Res also has the lowest Pearson correlation, which also implies the limited effect of IC-based measures in computing medicine semantic similarity. 
Wpath shows a slightly improvement over GADES and Res by adopting both structure information and semantic information of KGs. When we set Wpaths free parameter $\mathrm{k}=0.85$, Wpath can achieve its own highest correlation score.

The Hybrids method takes all aforementioned medicine-specific features into account to measure the semantic similarity. Its highest score among all baselines indicates the significance of the medicine-specific features.

Both Pearson and Spearman coefficient of MedSim are over 0.5, indicating that the prediction of our model has a high correlation with doctors judgment. Compared with other methods, MedSim can more effectively evaluate similarity. The results of the z-test also show that MedSim has a statistically significant improvement over baselines, since in each baseline $\mathrm{z}$ statistics are larger than 1.96 and p-values are below the significance level of 0.05. Experiment results also reveal that on the analytics and assessments of KG semantic/structure information, domain specific features need to be considered simultaneously.

\subsection{Feature Selection Comparison}

The prediction ability of each feature and feature combinations is measured in this section (Table 3).

Table 3. Comparison of feature performance.

\begin{tabular}{|l|l|l|l|}
\hline & Feature & Person & Spearman \\
\hline \multirow{3}{*}{ Single Feature } & MF & 0.407 & 0.389 \\
& HF & 0.159 & 0.150 \\
& SF & 0.339 & 0.258 \\
\hline \multirow{3}{*}{ Multiple Feature } & MF and HF & 0.551 & 0.489 \\
& MF and SF & 0.570 & 0.515 \\
& MF, HF and SF & $\mathbf{0 . 5 8 5}$ & $\mathbf{0 . 5 2 3}$ \\
\hline
\end{tabular}

For the single feature, the coefficient scores indicate that medicine-specific features yield a good performance without cooperating with other features .

The combinations of MF and HF and the combinations of MF and SF generally show much better performance than using these features separately, increasing the coefficients by at least $10 \%$. The best performance is obtained by the combination of all features, indicating that the proper combination of features can mine more information and improve the prediction performance.

There are four types of medicine-specific features adopted in our study, among which, the physiological effect based similarity with $21.3 \%$ Pearson coefficient outperforms other features. The using of each individual feature cannot yield a satisfactory result. Especially, the removal of the physiological effect based similarity weaken the prediction performance of model by decreasing the Pearson coefficient by $15.5 \%$. Through various pre-experimental results, we believe that the current combination of medicine-specific features is the one that is much helpful in the semantic similarity calculation of biomedicine. 


\subsection{Case Study}

To study the medicine substitution, we employ MedSim to predict the similarity scores between cefoperazone and other 51 antibiotics. All pairs containing cefoperazone are excluded from training set and considered as test set.

For the antibiotic cefoperazone, Table 4 presents its similar antibiotics whose similarity score is over 0.85 . Refer to 24, 25, the experiment results show that two antibiotics whose similarity scores over 0.85 can be replaced by each other under normal circumstances. We list the similar antibiotic names, provide the semantic similarity scores between antibiotics and cefoperazone evaluated by MedSim, and present the cases where they can replace each other.

Table 4. Parts of antibiotics similar can replace cefoperazone

\begin{tabular}{|l|l|l|}
\hline Similar Antibiotic & Score & Cause where the antibiotic can replace cefoperazone \\
\hline Cefoxitin & 0.865 & $\begin{array}{l}\text { Respiratory tract infections; Urinary tract infections; } \\
\text { Peritonitis; Septicemia; Gynecological infections; Bone, } \\
\text { joint, and soft tissue infections }\end{array}$ \\
\hline Cefepime & 0.864 & $\begin{array}{l}\text { Respiratory tract infections; Urinary tract infections; } \\
\text { Abdominal infections; Reproductive tract infections; } \\
\text { Bone, joint, and soft tissue infections }\end{array}$ \\
\hline Ceftriaxone & 0.860 & $\begin{array}{l}\text { Lower respiratory tract infection; Urinary tract infec- } \\
\text { tions; Complicated intra-abdominal infections; Infec- } \\
\text { tions in obstetrics and gynecology; Skin and soft tissue } \\
\text { infections; Meningitis }\end{array}$ \\
\hline Meropenem & 0.851 & $\begin{array}{l}\text { All infections of cefoperazone, but the has stronger effi- } \\
\text { cacy and wider antibacterial spectrums }\end{array}$ \\
\hline
\end{tabular}

Take cefoperazone and ceftriaxone as an example. The indication of cefoperazone is very close to ceftriaxone except disease caused by a few bacteria such as Pseudomonas aeruginosa. In the absence of susceptibility testing, doctors can choose either of them to treat most of Gram-negative bacteria infections, such as meningitis, pneumonia and bronchitis. Once the inventory of either is insufficient, our method can help doctors to find a most similar one for replacement.

Another example is cefoperazone and cefpime, both of which have good activity against Pseudomonas aeruginosa. However, the combined application of them cannot enhance the efficacy and is considered as drug abuse. Quantifying the similarity of antibiotics, such as listing antibiotics which have similar spectrum of bacterial susceptibility, may help improve public understanding that sometimes antibiotics combination should be avoided. Thus, medicine semantic similarity measure can ease the increasingly serious problem of antibiotic abuse.

Though meropenem can replace cefoperazone clinically, the semantic similarity score is slightly over 0.85 . The reason is that meropenem's indications far exceed cefoperazone. In other words, cefoperazone can not replace meropenem completely. The meropenem will be applied to replace cefoperazone only when the infective bacteria exceeded the antibacterial spectrum of cefoperazone or 
cefoperazone is ineffective. Otherwise, the replacement of cefoperazone with meropenem is clinically an abuse of antibiotics with higher antibacterial activity.

\section{Conclusion}

In this study, we propose MedSim, a novel semantic similarity method based on public well-established KGs and large-scale drug corpus. MedSim fully utilizes not only the structural and textual features from the KG but also medicinespecific features. MedSim produces statistically significant improvements over other methods. Examples of case study indicate that calculating the medicine semantic similarity owns a prospect in therapeutic substitution and decreasing the problem of drug abuse. The proposed method is extensible, reproducible and applicable to the KG-based similarity calculation in medical field. Assuming that a drug can be located in both a medical search engine and a bio-medical KG, all the features used in MedSim can be immediately obtained and used to measure other types of medicine in addition to antibiotics. All features used in MedSim can be obtained from the public knowledge source and the labeled dataset is now freely accessible, thus, our method can be conveniently reproduced. In the future, we explore the performance of MedSim in other types of medicine, such as sedative once we get the labeled respective drug dataset.

\section{References}

1. Hliaoutakis, A., Varelas, G., Petrakis, E. G. M., Milios E.: MedSearch: A Retrieval System for Medical Information Based on Semantic Similarity. Research and Advanced Technology for Digital Libraries Lecture Notes in Computer Science, 512515(2006).

2. Pedersen, T., Pakhomov, S. V., Patwardhan, S., Chute, C. G.: Measures of semantic similarity and relatedness in the biomedical domain. Journal of Biomedical In-formatics 40(3), 288-299(2007).

3. Resnik, P.: Semantic similarity in a taxonomy: an information-based measure and its application to problems of ambiguity in natural language. AI Access Foundation(1999).

4. Bollacker, K., Evans, C., Paritosh, P., Sturge, T., Taylor J.: Freebase. In: Proceedings of SIGMOD (2008).

5. Wishart, D. S., Feunang, Y. D., Guo, A. C., Lo, E. J., Marcu, A., Grant, J. R., Sajed, T., Johnson, D., Li, C., Sayeeda, Z., Assempour, N., Iynkkaran, I., Liu, Y., Maciejewski, A., Gale, N., Wilson, A., Chin, L., Cummings, R., Le, D., Pon, A., Knox, C., Wilson, M.: DrugBank 5.0: a major update to the DrugBank database for 2018, Nucleic Acids Research 46(D1), (2017).

6. Sun, Y., Han, J., Yan, X., Yu, PS., Wu, T.: PathSim: Meta Path-Based Top-K Similarity Search in Heterogeneous Information Networks. In: Proceedings of the Vldb Endowment 4(11), 992-1003 (2011).

7. Shi, C., Li, Y., Yu, P. S., Wu, B.: Constrained-meta-path-based ranking in heterogeneous information network. Knowledge and Information Systems 49(2), 719-747 (2016). 
8. Resnik, P.: Using information content to evaluate semantic similarity in a taxonomy. In: International Joint Conference on Artificial Intelligence, 448-453 (1995).

9. Mikolov, T., Sutskever, I., Chen, K., Corrado, G.S., Dean, J.: Distributed Representations of Words and Phrases and their Compositionality. Advances in neural information processing systems 26, 3111-3119 (2013).

10. Zhu, G., Iglesias, C.: Computing Semantic Similarity of Concepts in Knowledge Graphs. IEEE Transactions on Knowledge and Data Engineering 29(1), (2017).

11. Arup, C., Shrey, S., Pabitra, M., Cyril, S., Nandu, S.S., Muthusamy, C.: SimCat: an entity similarity measure for heterogeneous knowledge graph with categories. In: Proceedings of the Second ACM IKDD Conference on Data Sciences, 112-113 (2015).

12. Al-Mubaid, H., Nguyen, H.A.: A cluster-based approach for semantic similarity in the biomedical domain. In: 28th Annual International Conference of the IEEE, 2713-2717 (2006).

13. Traverso, I., Vidal, M.E., Kmpgen, B., Sure-Vetter, Y.: GADES: A Graph-based Semantic Similarity Measure. In: Proceedings of the 12th Interna-tional Conference on Semantic Systems, ACM, 101-104 (2016).

14. Pedersen, T., Pakhomov, S. V., Patwardhan, S., Chute, CG.: Measures of semantic similarity and relatedness in the biomedical domain. Journal of Biomedical Informatics 40(3), (2007).

15. Hliaoutakis, A.: Semantic similarity measures in MeSH ontology and their application to information retrieval on Medline. Master's thesis, (2005).

16. Kuhn, M., Letunic, I., Jensen, L. J., Bork, P.: The SIDER database of drugs and side effects. Nucleic Acids Research 44(D1), (2015).

17. Pathak, J., Chute, C. G.: Analyzing categorical information in two publicly available drug terminologies: RxNorm and NDF-RT. Journal of the American Medical Informatics Association 17(4), 432-439 (2010).

18. Canese, K., Weis, S.: PubMed: The bibliographic database. National Center for Biotechnology Information (2013).

19. Ho, I.W., Lee, C.T., Chen, P.W., Lo, Y.C.: Impact of Cumulative Antibiograms Sub-categorized by Origins of Infection Acquisition on the Selection of Empirical Antimicrobial Therapy. Journal of Biomedical And Laboratory Sciences 27(1), 10 18 (2015).

20. Hawkyard, C., Koerner, R.: The use of erythromycin as a gastrointestinal prokinetic agent in adult critical care: benefits versus risks authors' re-sponse. Journal of Antimicrobial Chemotherapy 61(1), 227-228 (2007).

21. Bryan, P., Rami A.R., Steven, S.: DeepWalk. In: Proceedings of SIGKDD (2014.

22. Gabrilovich, E., Markovitch S.: Computing semantic relatedness using wikipediabased explicit semantic analysis. International Joint Conference on Artifical Intelligence, 1606-1611 (2007).

23. Robertson, S.: The Probabilistic Relevance Framework: BM25 and Beyond. Foundations and Trends in Information Retrieval 3(4), 333-389 (2010).

24. Ho, P., Wong, S.: Reducing bacterial resistance with IMPACT-Interhospital Multidisciplinary Programme on Antimicrobial ChemoTherapy, Fourth Edition. Meteoritics And Planetaryence, 1-176, (2012).

25. Antibiotic guidelines, http://www.hopkinsmedicine.org/amp/guidelines/antibiotic \_guidelines.pdf . Last accessed 25 Jan 2018. 